\title{
Design of a Four-Branch Optical Power Splitter Based on Gallium-Nitride Using Rectangular Waveguide Coupling for Telecommunication Links
}

\author{
Retno W. Purnamaningsih (iD, ${ }^{1}$ Nji R. Poespawati, ${ }^{1}$ and Elhadj Dogheche ${ }^{2}$ \\ ${ }^{1}$ Department of Electrical Engineering, Universitas Indonesia, Kampus Baru UI, Depok, 16424, Indonesia \\ ${ }^{2}$ Université Polytechnique Hauts de France, CNRS IEMN DOAE, Valenciennes, France
}

Correspondence should be addressed to Retno W. Purnamaningsih; retno.wigajatri@ui.ac.id

Received 29 April 2019; Accepted 26 June 2019; Published 22 July 2019

Academic Editor: Paolo Colantonio

Copyright (c) 2019 Retno W. Purnamaningsih et al. This is an open access article distributed under the Creative Commons Attribution License, which permits unrestricted use, distribution, and reproduction in any medium, provided the original work is properly cited.

\begin{abstract}
This paper reports design of a simple four-branch optical power splitter using five parallel rectangular waveguides coupling in a gallium-nitride $(\mathrm{GaN})$ semiconductor/sapphire for telecommunication links. The optimisation was conducted using the 3D FDBPM method for long wavelength optical communication. The result shows that, at propagation length of $925 \mu \mathrm{m}$, the optical power input was successfully split into four uniform output beams, each with $24 \%$ of total input power. It is also shown that the relative output power distribution is almost stable through the C-band range. At the operating wavelength of $1.55 \mu \mathrm{m}$, the proposed power splitter has an excess loss lower than $0.2 \mathrm{~dB}$. This study demonstrates the opportunity to develop optical interconnections from UV-Visible to near IR wavelengths.
\end{abstract}

\section{Introduction}

Optical power splitters play an essential role as a passive component in optical communication. They are functioned for splitting and combining optical signals. Due to the rapid growth of data transmission, several splitter technologies have been proposed to expand data transmission capacity. So far many designs of optical power splitter have been developed, for example, using $\mathrm{Y}$ branch and multimode interference (MMI) structure on chip [1-3], photonic crystal [4-6], directional coupler [7], microring [8,9], and gratings [10].

Gallium-nitride semiconductor has important characteristics, such as mechanical hardness, high-temperature stability, and excellent transparency in telecommunication wavelength [11]. Therefore, III-nitride based devices may operate at much higher optical power levels. Those characteristics make it suitable for the harsh environment. Previous research indicated that III-nitrides have very lowtemperature sensitivity, especially in infrared wavelength regions [12]. This is another crucial advantage for applications in optical communications. Previous research [11] showed that small propagation loss occurred when gallium-nitride (GaN) with aluminium-nitride/gallium-nitride (AIN/GaN) buffer layer were integrated on sapphire substrates.

So far, the development of III-nitride based photonic devices has been focused on the light source [13-15] and photodetectors [16]. However, besides being developed as a light source and photodetectors, III-nitride based waveguide device design has also been developed. The design of the Y junction and MMI structure splitter using GaN/sapphire for long wavelength optical communication systems have been demonstrated $[17,18]$. A transferrable monolithic IIInitride photonic circuit for multifunctional optoelectronics has been developed [19]. A photonic circuit for visible light communication has also been reported [20]. GaN-based optical power splitter design for underwater optical wireless communication has also been demonstrated [21, 22]. However, to the best of our knowledge, the design of an optical power splitter based on GaN/sapphire using AlN/GaN buffer layer based on rectangular waveguide coupling for long wavelength telecommunication links has not yet been reported. 
This paper reports the design of a simple four-branch power splitter consisting of five identical parallel waveguides which are work based on mode coupling phenomena. The optical power splitter design and analysis relies on numerical calculations using commercial beam propagation software. The program is based on a finite difference beam propagation method (FD-BPM) to solve the paraxial approximation of the Helmholtz equation by applying the boundary conditions. The numerical experimental results of the proposed design are reported.

\section{Materials and Methods}

2.1. Operating Principles. If a waveguide is close enough to its adjacent waveguide, they become coupled and exchange power as a function of propagation direction. According to the scalar coupled mode theory, the field can be represented by a summation of the scalar modes of the individual waveguides. For configuration consists of two identical parallel waveguides, the effective indices of the symmetric and the antisymmetric normal modes can be approximated using conventional coupled mode theory as follows [23]:

$$
\begin{aligned}
& n_{s}=n_{e f f}+\kappa_{12} \\
& n_{a}=n_{e f f}-\kappa_{21}
\end{aligned}
$$

where the coupling coefficient is between waveguide 1 and waveguide 2 and vice versa; that is, $\kappa_{12}=\kappa_{21}=\kappa$ and $n_{\text {eff }}$ is the effective refractive index. The parameter describing the physics of the power transfer between waveguides is the coupling length, which is related to the difference between symmetric $\left(n_{s}\right)$ and antisymmetric $\left(n_{a}\right)$ refractive indices; that is,

$$
L_{c}=\frac{\lambda}{2\left(n_{s}-n_{a}\right)}
$$

where $\lambda$ is the wavelength.

2.2. The Proposed Design of Optical Power Splitter. The proposed structure of the waveguide is shown in Figure 1. The GaN structure layer consists of three layers, sapphire, the buffer layer, and GaN. Sapphire (yellow) is used as the wafer, AIN and AlN/GaN (white colour) are used as the buffer layer, GaN (brown colour) is used as the waveguide material, and air (no colour) is used as a cladding material. Figure 2 shows the proposed optical power splitter. The design is based on a conventional structure, five rectangular waveguides, where $\mathrm{Cg}$ is the coupling gap between two adjacent waveguides. $L_{i}$ is the length of the input waveguide and $L_{1}$ and $L_{2}$ are the length of waveguides 1 and 2, respectively, while $L_{3}$ and $L_{4}$ are the length of waveguide 3 to 4 accordingly. The basis of the design is to obtain a four uniform output power by optimising the waveguide parameter to enable power transfer that occurred between the adjacent waveguides. In this proposed design, the input waveguide is centred with the other four output waveguides. To obtain the desired output distribution, waveguide parameters, coupling gap, and

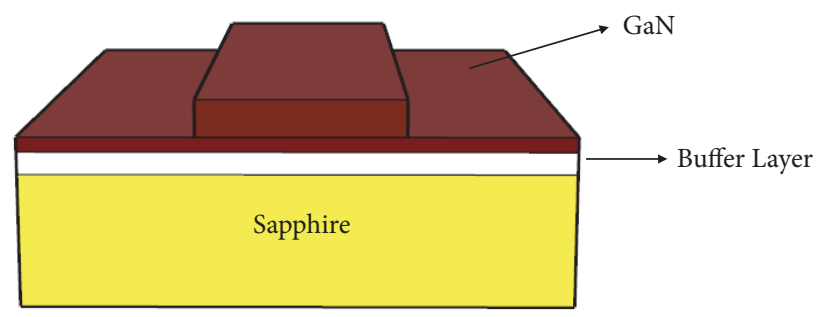

FIGURE 1: The schematic illustration of GaN structure layer in $x-y$ plane.

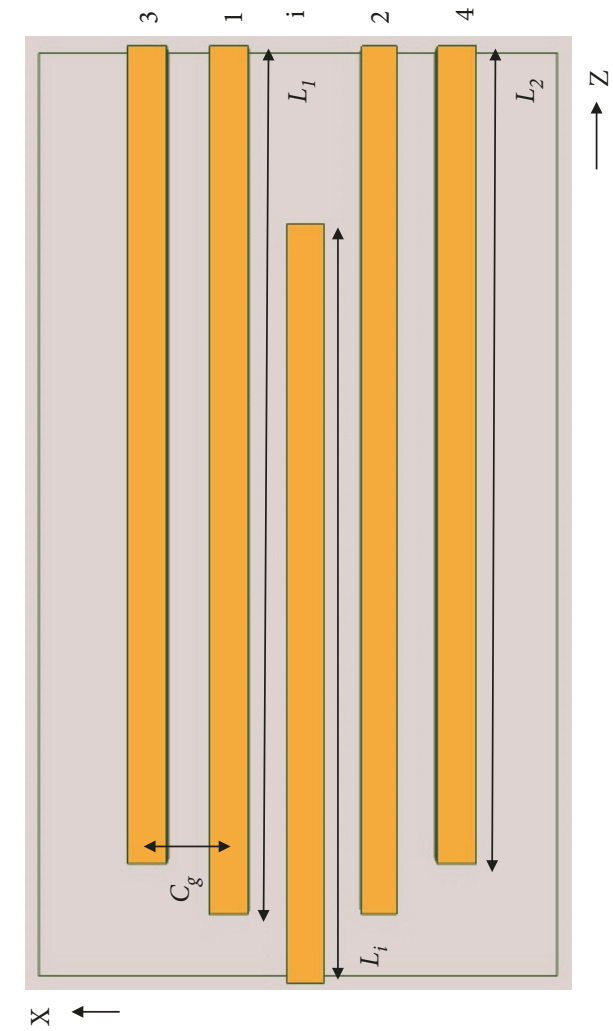

FIGURE 2: The schematic structure of the proposed four-branch optical power splitter in $x-z$ plane.

coupling length between the adjacent waveguides should be optimised.

In this design, the wurtzite structure of sapphire was used due to its temperature stability. Compared to other structures, this structure can increase the performances of the splitter. The effective refractive indices of the layers used were from the previous result [11], $\mathrm{nTE}=2.279 \pm 0.001$ and $\mathrm{nTM}=2.316$ \pm 0.001 . The first step optimised the width and the height of each waveguide to support only one mode of propagation. Next, the coupling gap and coupling length were optimised for TE polarisation. This step was conducted to obtain four separate single modes at the output ports. The numerical experiments were conducted by taking into account the losses and the uniformity of the optical power distribution. 
In this proposed design all the waveguides have the same width, thickness, and refractive index. For simplicity, the propagation constant of the fundamental propagation mode of all the waveguides can be assumed to have the same propagation constant, $\beta_{i}=\beta_{1}=\beta_{2}=\beta_{3}=\beta_{4}=\beta$. Since modes in all the waveguides are propagating in the same direction, the propagation constants are always positive, $\beta>$ 0 . Waveguides 1 and 2 are identical and symmetric, as well as waveguides 3 and 4 . Therefore, the amplitudes in waveguides 1 and 2 are the same, as well as waveguides 3 and 4 . However to achieve these conditions, the geometrical size of the structure should be optimised. In this design the power is injected only into the input waveguide; hence by applying the boundary conditions input amplitude is equal to 1 at propagation distance $\mathrm{z}=0$, while others are of zero value and the power transfer from input waveguide to the other four waveguides can be calculated. However, due to the complexity of the structure, the numerical analysis method had to be used.

2.3. Numerical Method for the Propagating Wave. To accurately design the photonic waveguides devices this requires numerical wave propagation techniques. So far, various numerical methods have been developed to solve Maxwell's equations. One of the most powerful techniques for studying the propagation of light in waveguide-based photonic devices is the beam propagation method (BPM). The BPM is very useful in solving light propagation in photonic waveguides of arbitrary shape. In BPM, a spatial mode is break down into a superposition of plane waves. Each wave propagates a small distance through the design of a waveguide structure. Next, to reconstruct the mode, the plane waves are then added back together. The general three-dimensional scalar Helmholtz equation is as follows [24]:

$$
\nabla^{2} E(x, y, z)+k_{0}^{2} n^{2}(x, y, z) E(x, y . z)=0
$$

where $k_{0}$ is the wave number in vacuum. Using the slowly varying envelope approximation the electric field $E(x, z)$ is divided into the slowly varying envelope function $\Phi(x, y, z)$ and a spatially very fast oscillating phase term $\exp (-j \beta z)$ as follows:

$$
E(x, y, z)=\Phi(x, y, z) \exp (-j \beta z)
$$

Here, $\beta=k n_{\text {eff }} ; n_{\text {eff }}$ is the effective refractive index. In actual calculations, this value is a priori unknown and can be set to the refractive index of the substrate or cladding. Next, by substituting (5) into (4) and dividing both sides of the resultant equation by $\exp (-j \beta z)$ we can obtain

$$
\frac{\partial^{2} \Phi}{\partial z^{2}}-2 j \beta \frac{\partial \Phi}{\partial z}+\nabla_{\perp}^{2} \Phi+\left(k_{0}^{2} n^{2}-\beta^{2}\right) \Phi=0
$$

where $\nabla_{\perp}=\partial^{2} / \partial x^{2}+\partial^{2} / \partial y^{2}$ is the Laplacian in the lateral directions and (6) can also be written as

$$
2 j \beta \frac{\partial \Phi}{\partial z}-\frac{\partial^{2} \Phi}{\partial z^{2}}=\nabla_{\perp}^{2} \Phi+k_{0}^{2}\left(\varepsilon_{r}-n_{e f f}\right) \Phi
$$

where $\varepsilon_{r}=n^{2}$. Next, by neglecting second derivatives, that is, $\partial^{2} \Phi / \partial z^{2}=0$, (7) is reduced to

$$
2 j \beta \frac{\partial \Phi}{\partial z}=\nabla_{\perp}^{2} \Phi+k_{0}^{2}\left(\varepsilon_{r}-n_{e f f}\right) \Phi
$$

Here, $\partial^{2} \Phi / \partial z^{2}$ has been neglected by assuming $\left|\partial^{2} \Phi / \partial z^{2}\right| \ll$ $2 j \beta|\partial \Phi / \partial z|$. This approximation is called the Fresnel approximation or the paraxial approximation.

For TE mode, $\partial \Phi / \partial y=0$. The Fresnel equation can be written as follows:

$$
2 j \beta \frac{\partial \Phi}{\partial z}=\partial_{x}^{2} \Phi+k_{0}^{2}\left(\varepsilon_{r}-n_{e f f}\right) \Phi
$$

Equation (9) can be solved using a finite difference method. The region of interest is divided into a grid of nodes. The selection of the original grid size should be small enough so that the solution converges. The discretisation of the $x$ and $z$ coordinates is according to

$$
\begin{aligned}
x & =p \triangle x \\
\text { and } z & =l \triangle z
\end{aligned}
$$

where $p$ and $l$ are integers, $\Delta x$ and $\Delta z$ denote the calculation steps in the $x$-axis and $z$-axis directions, respectively, and the propagation step distance $x$ as well as $z$ can be different for each propagation step. Mostly the use of equidistant discretization for the $x$ - and $z$-direction is used. The differential equation of (9) can be discretized in $x$ direction; that is, the terms of right hand are expressed as follows:

$$
\begin{aligned}
\frac{\partial^{2} \Phi}{\partial x^{2}} & =\frac{1}{\Delta x}\left(\frac{\phi_{p+1}-\phi_{p}}{(\Delta x)}-\frac{\phi_{p}-\phi_{p-1}}{(\Delta x)}\right) \\
& =\frac{\phi_{p+1}-2 \phi_{p}+\phi_{p-1}}{(\Delta x)^{2}} \\
k_{0}^{2}\left(\varepsilon_{r}-n_{e f f}^{2}\right) \Phi & =k_{0}^{2}\left(\varepsilon_{r}(p)-n_{e f f}^{2}\right) \Phi_{p}
\end{aligned}
$$

The discretisation of the left-hand side of (9) in the $z$ direction is presented as follows:

$$
\begin{gathered}
2 j \beta \frac{\phi_{p}^{l+1}-\phi_{p}^{l}}{(\Delta z)}-\frac{\phi_{p}-\phi_{p-1}}{(\Delta x)} \frac{\partial \Phi}{\partial z} \\
=\partial_{x}^{2} \Phi+k_{0}^{2}\left(\varepsilon_{r}-n_{e f f}\right) \Phi
\end{gathered}
$$

The subscripts $p$ and $l$ are indices of the sampling points along $x$-direction and $z$-direction, accordingly. The number of grid points along the $x$-axis and $z$-axis directions is $P(p=1,2, \ldots$, $P)$ and $L(l=1,2, \ldots, L)$, respectively. The term $\phi_{p}^{l}$ denotes the discrete values of the field at $x_{p}=p \Delta x$ and $z_{l}=l \Delta z$. 


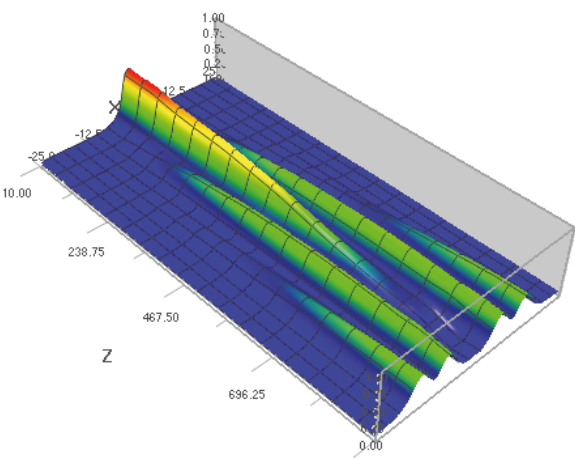

(a)

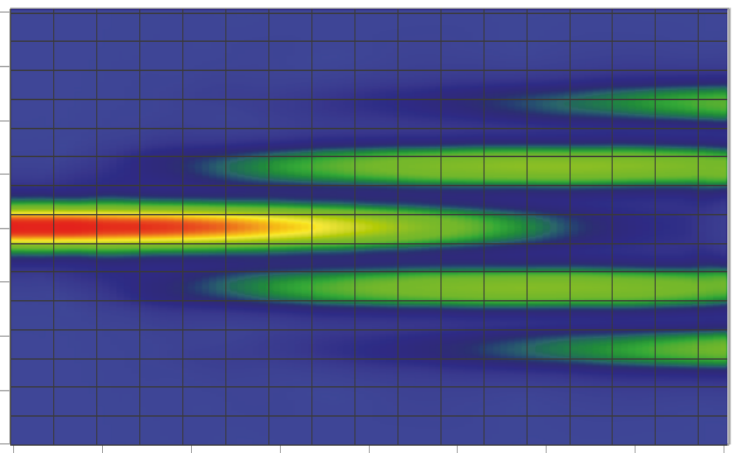

(b)

FIgURE 3: 3D (a) and 2D (b) optical field distribution of the proposed optical power splitter along $x-z$ plane.

Finally, the finite difference expression of $E_{y}(x, y, z)$ for TE mode is as follows:

$$
\begin{aligned}
-\alpha_{w}^{l+1} & \phi_{p-1}^{l+1} \\
& +\left\{-\alpha_{x}^{l+1}+\frac{4 j \beta}{\Delta z}-k_{0}^{2}\left[\varepsilon_{r}^{l+1}(p)-n_{e f f}^{2}\right]\right\} \phi_{p}^{l+1} \\
& -\alpha_{\varepsilon}^{l+1} \phi_{p+1}^{l+1} \\
= & -\alpha_{w}^{l} \phi_{p-1}^{l}+\left\{\alpha_{x}^{l}+\frac{4 j \beta}{\Delta z}+k_{0}^{2}\left[\varepsilon_{r}^{l}(p)-n_{e f f}^{2}\right]\right\} \phi_{p}^{l} \\
& -\alpha_{\varepsilon}^{l} \phi_{p+1}^{l}
\end{aligned}
$$

where $\alpha_{w}=1 /\left(\Delta x^{2}\right), \alpha_{\varepsilon}=1 /\left(\Delta x^{2}\right)$, and $\alpha_{\varepsilon}=1 /-\left(\Delta x^{2}\right)$

Once the initial electric field distribution $\phi_{0}^{l}(l=1,2, \ldots$, $L)$ at the input position is given, the electric field profile $\phi_{p}^{l}(p$ $=1,2, \ldots, P)$ can be calculated at any distance $z_{i}$ by solving the tridiagonal system of linear equations.

\section{Results and Discussion}

The numerical experiments were conducted using OptiBPM software based on a slowly varying envelope approximation combined with MATLAB software. The field propagation calculations through the structure were carried out using the finite difference beam propagation method (FD-BPM), which is suitable for the proposed design. In these numerical experiments, we used the TE polarised field; therefore, a semivectorial FD-BPM is applied. To obtain the best geometrical value of the proposed splitter, first, the width of each waveguide was varied from $2 \mu \mathrm{m}$ up to $5 \mu \mathrm{m}$; then the relative optical power was observed. It was found that the 4 $\mu \mathrm{m}$ waveguide width gave the highest relative optical power at 0.99 . Secondly, by employing this width value, the best thickness of each waveguide was optimised by varying the waveguide thickness from $0.5 \mu \mathrm{m}$ up to $5 \mu \mathrm{m}$ and observed the relative output power. It was shown that $4 \mu \mathrm{m}$ thickness gave the highest optical power at 0.99 . Therefore, the $4 \mu \mathrm{m}$ width and $4 \mu \mathrm{m}$ thickness of each waveguide were chosen. The following was the optimisation of the gap between adjacent waveguides by observing power loss for various gaps, starting at $0.5 \mu \mathrm{m}$ up to $5 \mu \mathrm{m}$. The result showed that $3 \mu \mathrm{m}$ of gap gave the lowest power loss, at 0.98 a.u. Finally the optimum length of each waveguide was defined based on analytic calculation of the coupling length and the coupling coefficient.

By solving (1) combined with semivector BPM simulations, the value of coupling length $L_{c}$ and coupling length $\kappa$ can be obtained. The calculated value of $L_{c}$ obtained was 254.3 $\mu \mathrm{m}$ with $\kappa=0.0062 \mu \mathrm{m}^{-1}$. Using this result, the length of each waveguide was optimised to obtain the uniform distribution and single mode propagation in each waveguide. It was found that the best length for the input waveguide is $780 \mu \mathrm{m}$, the output waveguides 1 and 2 are $875 \mu \mathrm{m}$, and waveguides 3 and 4 are $825 \mu \mathrm{m}$ accordingly. It can be noticed that a coupling gap of $7 \mu \mathrm{m}$ is needed to obtain an almost equal power transfer between the waveguides. The total length and width of the power splitter are $925 \mu \mathrm{m}$ and $32 \mu \mathrm{m}$, respectively. This size gives the minimum imbalance and lowest excess loss at $1.55 \mu \mathrm{m}$. In Figures 3(a) and 3(b), the map of optical field distribution through the proposed structure for both $3 \mathrm{D}$ and $2 \mathrm{D}$ is, respectively, displayed. The different colours red, yellow, and green represent the level of the optical field. It is shown that the incoming power is split into an almost four equal intensities (green colour) in each output branch. It is also shown that a strong confinement (red colour) was obtained inside the GaN layer.

Figure 4 shows the mode propagation for $\lambda=1550 \mu \mathrm{m}$ at several propagation distances, $\mathrm{z}=0, \mathrm{z}=675 \mu \mathrm{m}$, and $\mathrm{z}=925$ $\mu \mathrm{m}$. It should be noted that the optical power begins to split at $\mathrm{z}=675 \mu \mathrm{m}$; it is then divided into four optical powers at $\mathrm{z}$ $=925 \mu \mathrm{m}$.

Next, to observe the power loss at the telecommunication wavelength throughout the proposed power splitter, the total relative optical power at $\lambda=1550 \mathrm{~nm}$ along the propagation $z$-axis was analysed. The results showed that the total relative power along the proposed splitter was stable at 0.98 a.u., as shown in Figure 5. Furthermore, the power exchange between waveguides was explored. Figure 6 shows the power exchange between input waveguide and waveguide 1. It shows the power exchange periodically between input waveguide 


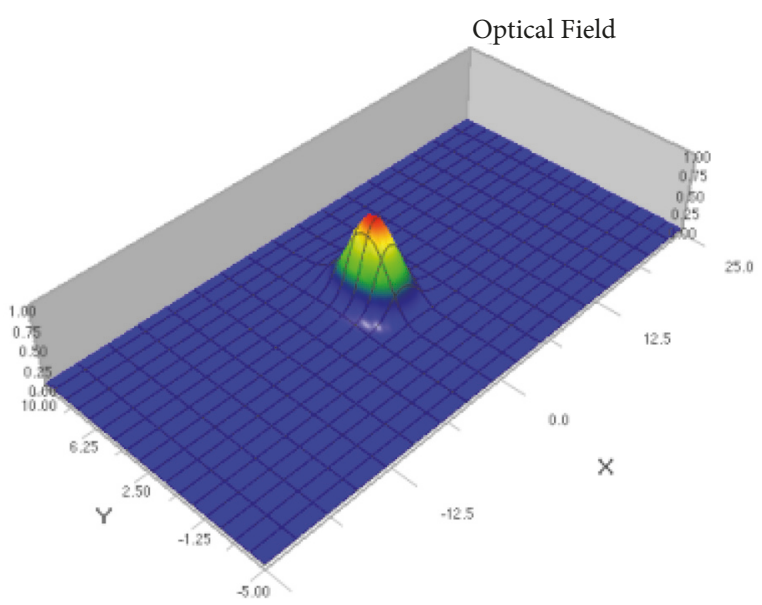

(a) $z=0$

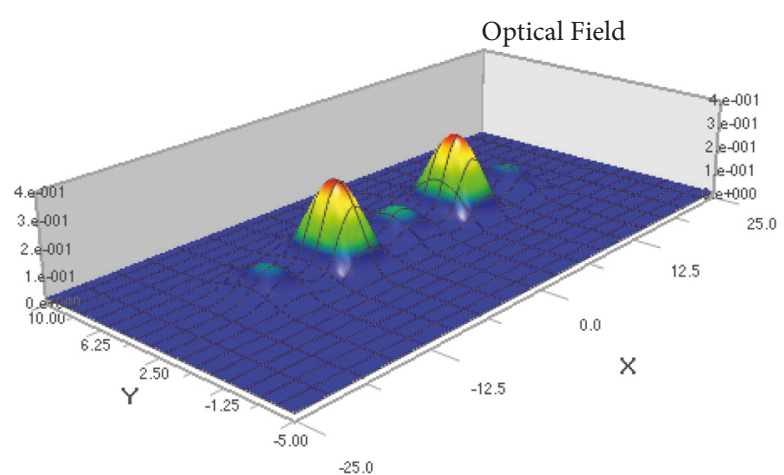

(b) $z=675 \mu \mathrm{m}$

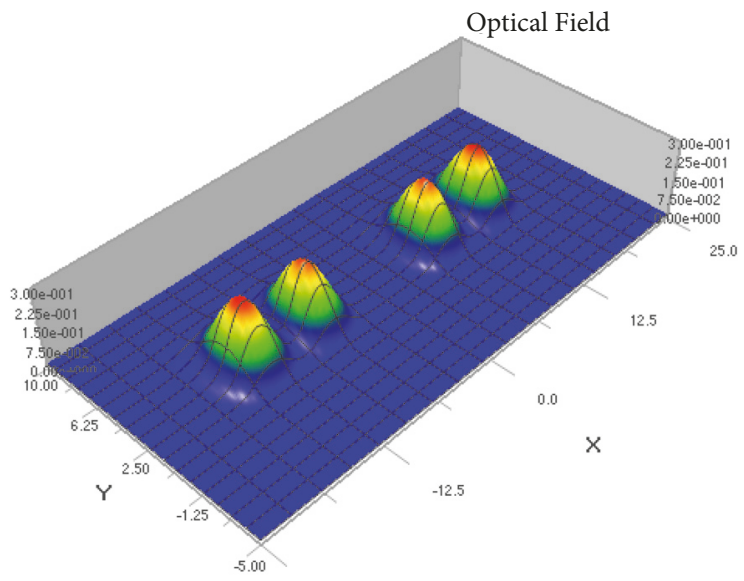

(c) $z=925 \mu \mathrm{m}$

FiguRE 4: 3D normalized optical intensity field distribution at the output ports for various propagation distances, (a) $z=0$, (b) $z=675 \mu \mathrm{m}$, and (c) $z=925 \mu \mathrm{m}$.

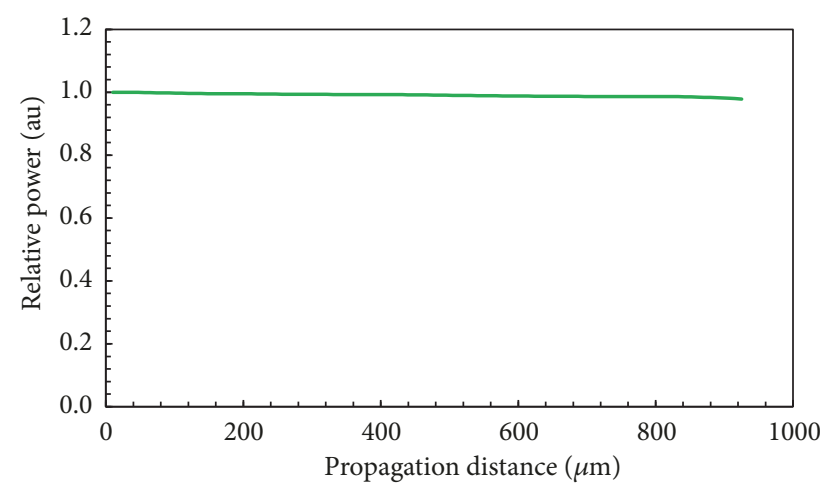

FIGURE 5: The total relative power throughout the proposed power splitter at $\lambda=1550 \mathrm{~nm}$.

and waveguide 1 . At a distance of $\mathrm{z}=925 \mu \mathrm{m}$, the power is completely transferred from input waveguide to waveguide 1 , while at a distance of $\mathrm{z}=550 \mu \mathrm{m}$ half the input power is transferred. This exchange power mechanism is due to mode coupling that occurred between the waveguides.

To obtain the complete output power distribution, the power transfer mechanism between waveguides was also investigated. The result, among others, is shown in Figure 7, which displayed the optical power transfer between waveguides 1 and 3. It should be noted that the optical input power faded away at $z=780 \mu \mathrm{m}$, due to the power transferred to waveguides 1 and 2. By investigating the total relative power throughout the proposed power splitter (Figure 5) and relative power for each waveguide throughout the propagation axis (Figure 7), it can be obtained that the optical power of each output port has an almost uniform splitting ratio of about $24 \%$ at each port.

Furthermore, the tolerance value for the structure to anticipate the fabrication error was investigated by varying the coupling gap between the adjacent waveguides. The coupling gap was varied from $6 \mu \mathrm{m}$ up to $8 \mu \mathrm{m}$. The results, among others, are shown in Figure 8. It should be noted that the tolerance range of the coupling gap value should be under $0.5 \mu \mathrm{m}$ from the best value of $8 \mu \mathrm{m}$. 


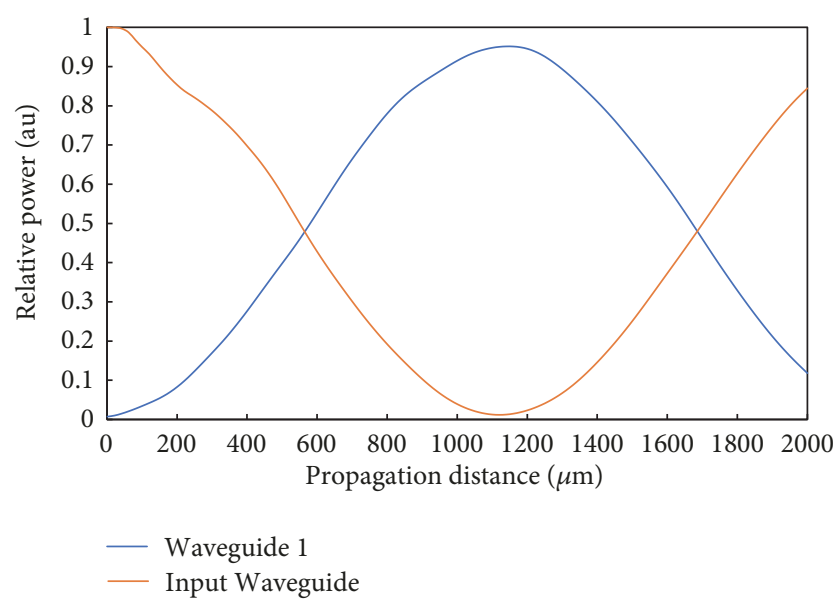

FIGURE 6: Exchange of power between input waveguide and waveguide 1.

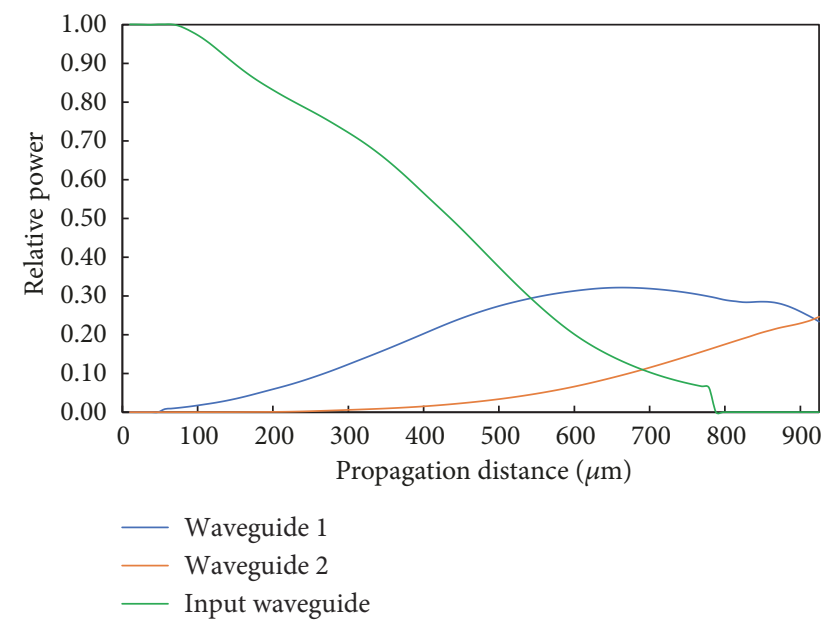

FIGURE 7: The relative power as a function of propagation distance $(z)$ for waveguides 1 and 3 .

Next, performances of the power splitters are expressed in excess loss, power imbalance, and transmission coefficient. Excess loss is the amount of power lost due to reflection and other material absorption properties. The excess loss was calculated as follows:

$$
\text { Excess Loss }=-10 \log \left(\frac{\mathrm{P}_{\text {out }}}{\mathrm{P}_{\text {in }}}\right)
$$

where $\mathrm{P}_{\text {out }}$ and $\mathrm{P}_{\text {in }}$ are the total optical output power and the optical input power, respectively. The amount of power imbalance in a splitter is given by the ratio of maximum output power to the minimum output power as defined in (16).

$$
\text { Imbalance }=-10 \log \left(\frac{\mathrm{P}_{\min }}{\mathrm{P}_{\max }}\right)
$$

where $\mathrm{P}_{\max }$ is the highest optical power and $\mathrm{P}_{\min }$ is the lowest optical power at the four output ports. By using (15) and (16), excess loss and power imbalance of the splitter across
$100 \mathrm{~nm}$ spectrum of wavelength were calculated at the best geometrical value of the proposed power splitter. The results are presented in Figures 9 and 10 accordingly. Figure 9 shows that, at $\lambda=1550 \mathrm{~nm}$, the power splitter has an excess loss of $0.18 \mathrm{~dB}$. It can be noted that the excess loss differences varied $\pm 0.016 \mathrm{~dB}$ across $100 \mathrm{~nm}$ spectrum of wavelength. At C-band range (1530-1565 nm), the excess loss varied from $0.18 \mathrm{~dB}$ up to $0.19 \mathrm{~dB}$. Due to the guiding mechanism based on mode coupling, it does not involve an interference effect. Therefore, the splitter presents low wavelength sensitivity.

Figure 10 presents the power imbalance of the splitter as a function of wavelength. It can be noticed that, at the operating wavelength, the power imbalance yields are $0.23 \mathrm{~dB}$. Across the C-band range, the power imbalance varied from $0.23 \mathrm{~dB}$ up to $0.59 \mathrm{~dB}$. The excess loss and power imbalance of the proposed design are slightly higher in comparison to other optical power splitter designs on slot silicon-gallium nitride waveguide [25].

Next, the transmission coefficient for each output waveguide at the C-band range was observed by calculating it using (17) as follows:

$$
\text { Transmission Coefficient }=-10 \log \left(\frac{\mathrm{P}_{\mathrm{ow}}}{\mathrm{P}_{\mathrm{in}}}\right)
$$

where $\mathrm{P}_{\mathrm{ow}}$ and $\mathrm{P}_{\text {in }}$ are the power of each output port and power at input port, respectively.

The result is displayed in Figure 11.

Figure 11 displays the transmission coefficient of the splitter across $100 \mathrm{~nm}$ spectrum of wavelength. It is found that the transmission coefficient of waveguides 1 and 2 increases from $5.9 \mathrm{~dB}$ up to $7.1 \mathrm{~dB}$, while waveguides 3 and 4 decrease from $6.8 \mathrm{~dB}$ to $5.8 \mathrm{~dB}$. At the operating wavelength 1.55 $\mu \mathrm{m}$, the power splitter divided the input power close to the optimum values.

Furthermore, we investigate the output power of the proposed design across the whole C-band by calculating the normalized transmitted power for $\lambda=1500-1600 \mathrm{~nm}$ using FD-BPM method. The result is displayed in Figure 12. It should be noted that the normalized transmitted power varies about $0.8 \%$, from 0.980 to 0.972 over $100 \mathrm{~nm}$ spectrum scope.

In the range of C-band (1530-1565 nm), the relative output power distribution varies from 0.980 to 0.977 . It means that the design is almost stable through this range. Therefore, it opens an opportunity for future technology.

\section{Conclusions}

A simple $1 \times 4$ optical power splitter consisting of five parallel rib waveguides based on mode coupling using $\mathrm{GaN} /$ sapphire was studied. The optimisation was conducted using FD-BPM and MATLAB at long wavelength optical communication $\lambda$ $=1550 \mathrm{~nm}$. The results show that the proposed structure gives an excess loss of $0.18 \mathrm{~dB}$ with a power imbalance of $0.23 \mathrm{~dB}$. It is also shown that, at the C-band range, the transmission coefficient of waveguides 1 and 2 increases from $5.9 \mathrm{~dB}$ up to $7.1 \mathrm{~dB}$, while waveguides 3 and 4 decrease from $6.8 \mathrm{~dB}$ to $5.8 \mathrm{~dB}$. The design is simple; therefore, it is easy to fabricate and can be further developed to be applied in optical communication technology. 


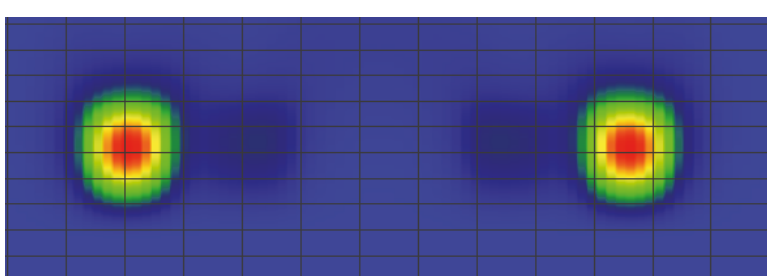

(a)

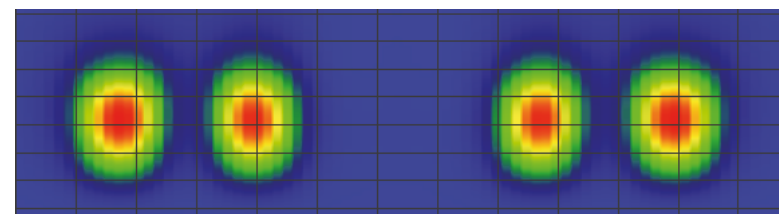

(b)

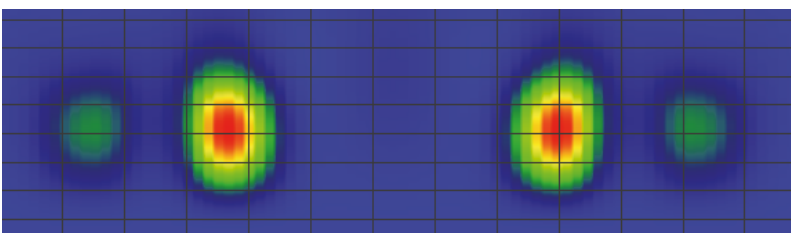

(c)

Figure 8: Optical fields profile at the output ports for various coupling gaps (a) $6.5 \mu \mathrm{m}$ (b) $7 \mu \mathrm{m}$ and (c) $7.5 \mu \mathrm{m}$.

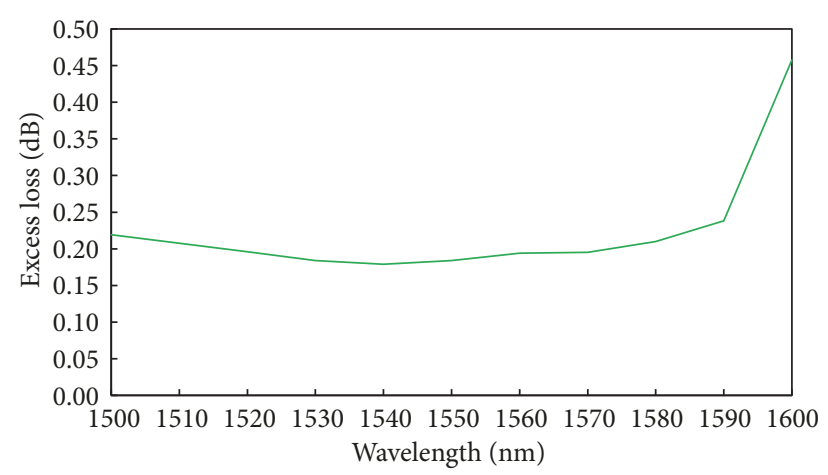

Figure 9: The excess loss of the TE mode in the four-branch optical power splitter.

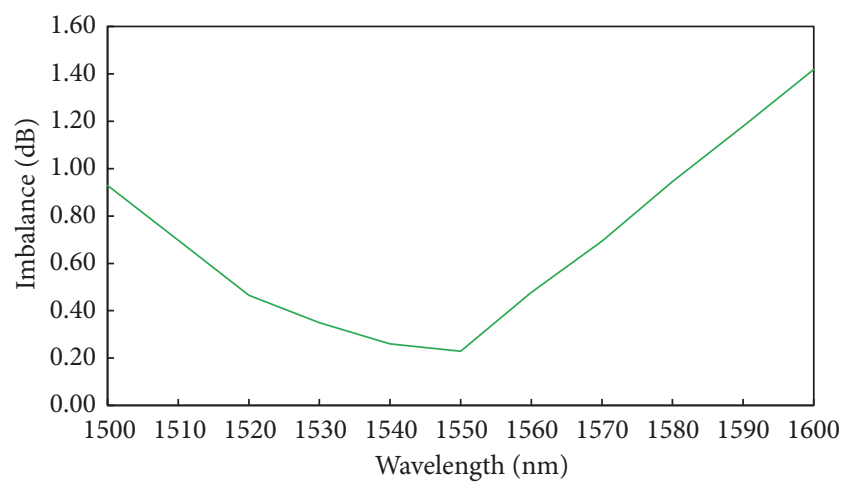

FIGURE 10: The power imbalance of the TE mode in the four-branch optical power splitter.

\section{Data Availability}

The data used to support the findings of this study are available from the corresponding author upon request.

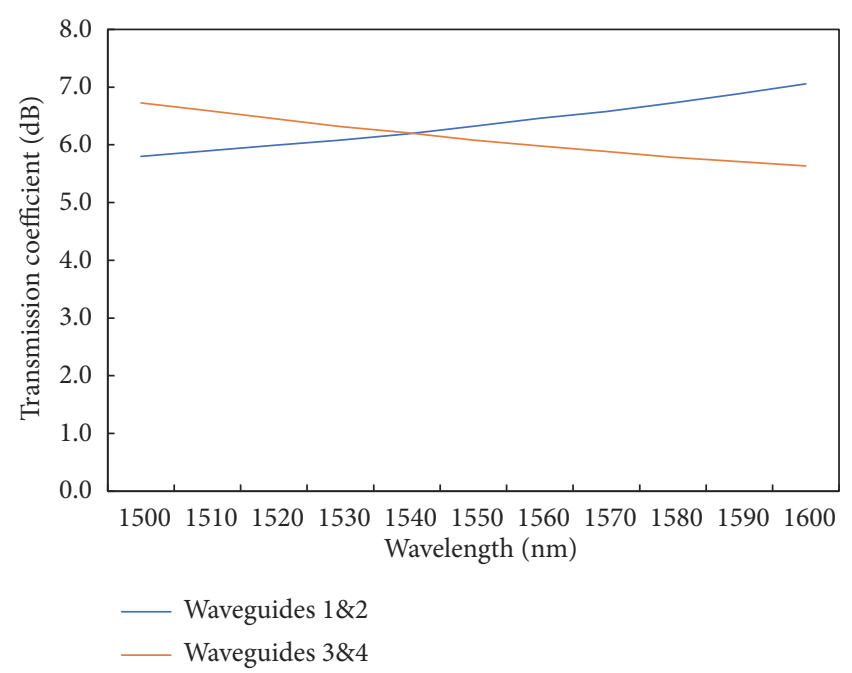

FIGURE 11: The transmission coefficient of each output port for the TE mode in the four-branch optical power splitter.

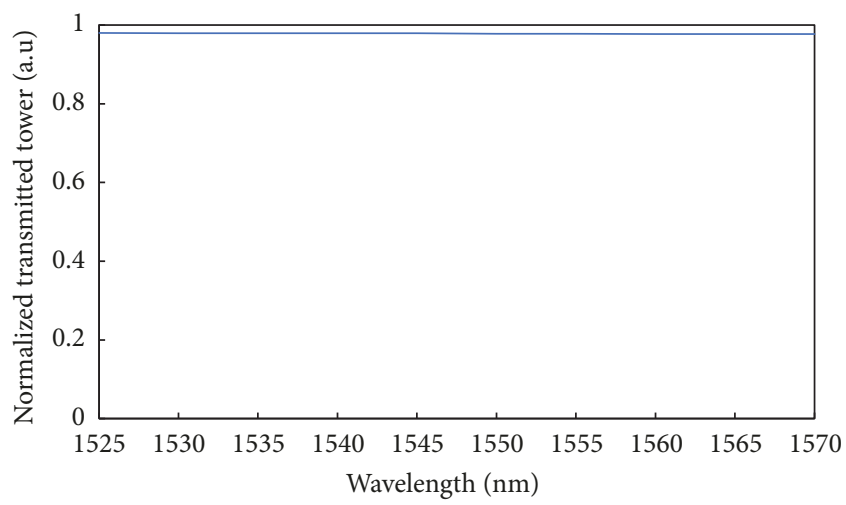

FIGURE 12: Relative power distribution for the wavelength range from 1500 up to $1600 \mathrm{~nm}$. 


\section{Additional Points}

We have changed the operating wavelength for telecommunication links purposes and investigated the effect of the coupling gap to the profile of the optical field at the output ports. Besides, the waveguide parameters and stability of relative optical power in the $\mathrm{C}$-band range were also observed.

\section{Disclosure}

This paper reports an extensive work of our previous paper presented in ICETAS2018 "A GaN/sapphire 1×4 Optical Power Splitter Using Five Rectangular Waveguides for Underwater Application.”

\section{Conflicts of Interest}

The authors declare that they have no conflicts of interest regarding the publication of this paper.

\section{Acknowledgments}

This research is supported by Research Grant 2019 (No. NKB-788/UN2.R3.1/HKP.05.00/2019) from RISTEK DIKTI, Indonesia.

\section{References}

[1] N. Pendam and C. P. Vardhani, "Design, simulation \& optimization of 3D low-loss asymmetrical Y-branch optical power splitter on SOI platform," in Proceedings of the 2016 International Conference on Electrical, Electronics, and Optimization Techniques, ICEEOT 2016, pp. 755-757, India, March 2016.

[2] C. Li, X. Li, X. Qiu, and Y. Xi, "A novel planar waveguide supermultiple-channel optical power splitter," Journal of Lightwave Technology, vol. 33, no. 24, pp. 5019-5024, 2015.

[3] K. Kim, Q. V. Vuong, Y. Kim, and M. Kwon, "Compact silicon slot waveguide intersection based on mode transformation and multimode interference," IEEE Photonics Journal, vol. 9, no. 6, Article ID 4502910, pp. 1-10, 2017.

[4] L. Nikolaevsky, T. Shchori, and D. Malka, "Modeling a $1 \times 8$ MMI green light power splitter based on gallium-nitride slot waveguide structure," IEEE Photonics Technology Letters, vol. 30, no. 8, pp. 720-723, 2018.

[5] S. Singh and K. Singh, "Design of an integrated multi-arm power splitter using photonic crystal waveguide," Optics, vol. 145, no. 9, pp. 495-502, 2017.

[6] D. Malka and A. Peled, "Power splitting of $1 \times 16$ in multicore photonic crystal fibers," Applied Surface Science, vol. 417, no. 09, pp. 34-39, 2017.

[7] E. Peter, A. Thomas, A. Dhawan, and S. R. Sarangi, "Active microring based tunable optical power splitters," Optics Communications, vol. 359, pp. 311-315, 2016.

[8] T. Spuesens, S. Pathak, M. Vanslembrouck, P. Dumon, and W. Bogaerts, "Grating couplers with an integrated power splitter for high-intensity optical power distribution," IEEE Photonics Technology Letters, vol. 28, no. 11, pp. 1173-1176, 2016.
[9] J. Xiao and Z. Guo, "Ultracompact polarization-insensitive power splitter using subwavelength gratings," IEEE Photonics Technology Letters, vol. 30, no. 6, pp. 529-532, 2018.

[10] S. C. Jain, M. Willander, J. Narayan, and R. van Overstraeten, "III-nitrides: growth, characterization, and properties," Journal of Applied Physics, vol. 87, no. 3, pp. 965-1006, 2000.

[11] A. Stolz, E. Cho, E. Dogheche et al., "Optical waveguide loss minimized into gallium nitride based structures grown by metal organic vapor phase epitaxy," Applied Physics Letters, vol. 98, no. 16, Article ID 161903, 2011.

[12] U. Tisch, B. Meyler, O. Katz, E. Finkman, and J. Salzman, "Dependence of the refractive index of AlxGal-xN on temperature and composition at elevated temperatures," Journal of Applied Physics, vol. 89, no. 5, pp. 2676-2685, 2001.

[13] D. L. Becerra, D. A. Cohen, R. M. Farrell, S. P. DenBaars, and S. Nakamura, "High efficiency semipolar III-nitride lasers for solid state lighting," in Proceedings of the 2016 International Semiconductor Laser Conference, ISLC 2016, Japan, September 2016.

[14] R. Horng, S. Chuang, C. Tien, S. Lin, and D. Wuu, "High performance GaN-based flip-chip LEDs with different electrode patterns," Optics Express, vol. 22, no. S3, p. A941, 2014.

[15] Y. Zhang, W. Q. Qin, X. Zhu, J. Xiao, and C. Yao, "The study on LED droop of flip-chip GaN LED," in Proceedings of the 2018 19th International Conference on Electronic Packaging Technology (ICEPT), pp. 1047-1050, Shanghai, China, August 2018.

[16] R. Velazquez, A. Aldalbahi, M. Rivera, and P. Feng, "Fabrications and application of single crystalline $\mathrm{GaN}$ for highperformance deep UV photodetectors," AIP Advances, vol. 6, no. 8, Article ID 085117, 2016.

[17] R. W. Purnamaningsih, I. Saraswati, N. R. Poespawati, and E. Dogheche, "Design of gan-based low-loss y-branch power splitter," Makara Journal of Technology, vol. 18, no. 3, pp. 101106, 2015.

[18] R. W. Purnamaningsih, N. R. Poespawati, and E. Dogheche, "III-nitride semiconductors based optical power splitter device design for underwater application," International Journal of Electrical and Computer Engineering, vol. 8, no. 5, pp. 38663874, 2018.

[19] Z. Shi, X. Gao, J. Yuan et al., "Transferrable monolithic IIInitride photonic circuit for multifunctional optoelectronics," Applied Physics Letters, vol. 111, no. 24, 2017.

[20] Y. Jiang, Z. Shi, X. Gao, J. Yuan, S. Zhang, and Y. Wang, "Monolithic III-nitride photonic circuit for multifunctional visible light communication," in Proceedings of the 2017 IEEE/CIC International Conference on Communications in China (ICCC Workshops), pp. 1-2, Qingdao, October 2017.

[21] R. W. Purnamaningsih, M. Hamidah, D. Fithriaty, M. R. Gumelar, N. R. Poespawati, and E. Dogheche, "A GaN/sapphire $1 \times 4$ optical power splitter using five rectangular waveguide for underwater application," in Proceedings of the 5th IEEE International Conference on Engineering Technologies and Applied Sciences, ICETAS 2018, Bangkok, Thailand, November 2018.

[22] R. W. Purnamaningsih, N. R. Poespawati, T. Abuzairi, and E. Dogheche, "An optical power divider based on mode coupling using gan/al2o3 for underwater communication," Photonics, vol. 6, no. 2, p. 63, 2019.

[23] W. P. Huang, "Coupled Mode theory for optical waveguide: an overview," Journal of the Optical Society of America A, vol. 11, no. 3, pp. 963-983, 1994. 
[24] K. Kawano and T. Kitoh, Fundamentals of Optical Waveguide, John Wiley \& Sons, Inc, 2001.

[25] D. Malka, Y. Danan, and Z. Zalevsky, "Slot silicon-gallium nitride waveguide realizing $1 \times 4$ optical power splitter," in Proceedings of the 2016 IEEE International Conference on the Science of Electrical Engineering (ICSEE), pp. 1-5, Eilat, Israel, November 2016. 


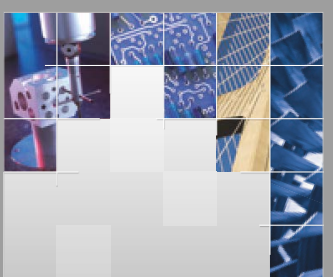

\section{Enfincering}
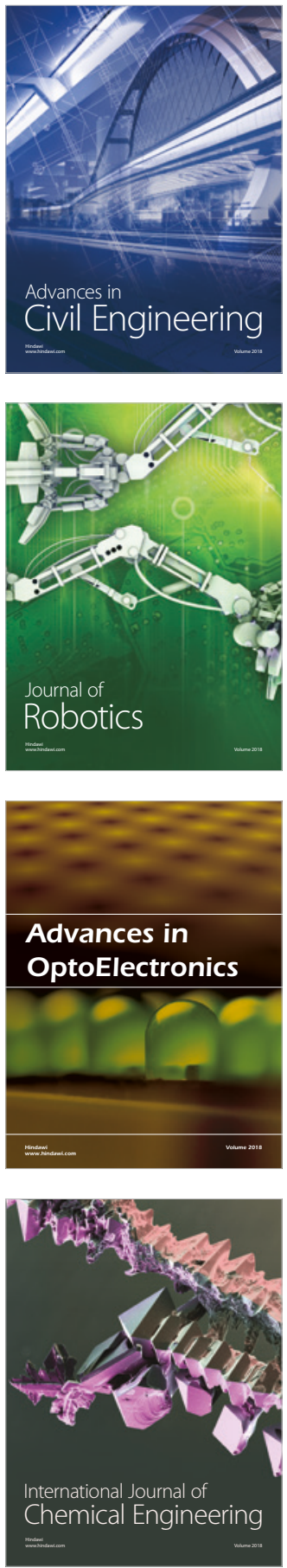

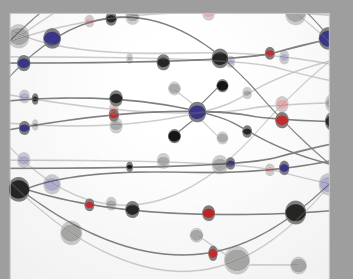

\section{Rotating \\ Machinery}

The Scientific World Journal

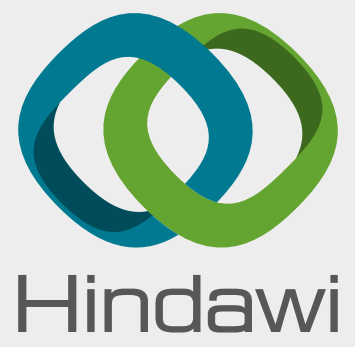

Submit your manuscripts at

www.hindawi.com
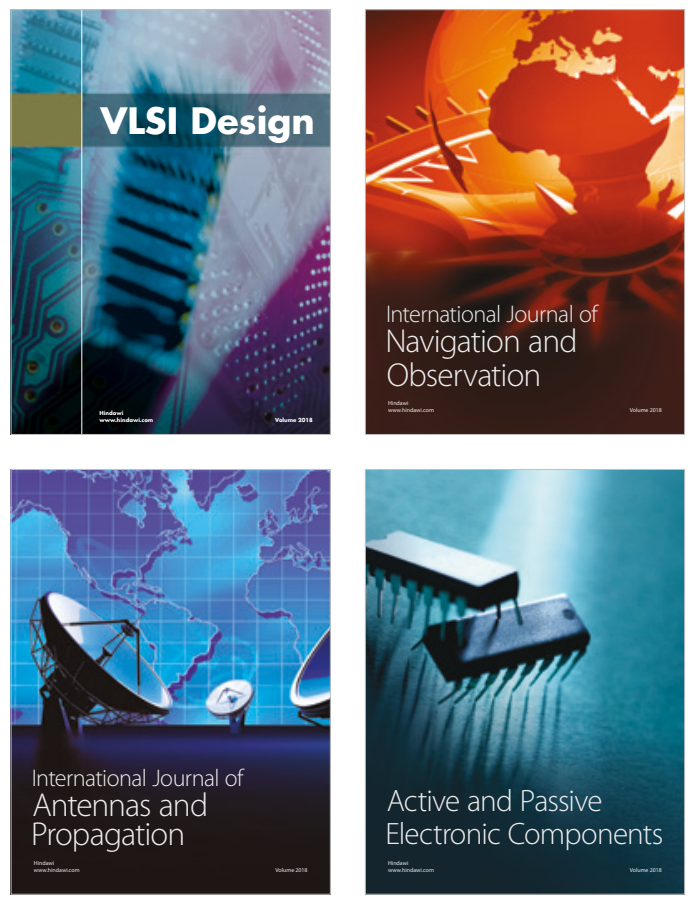
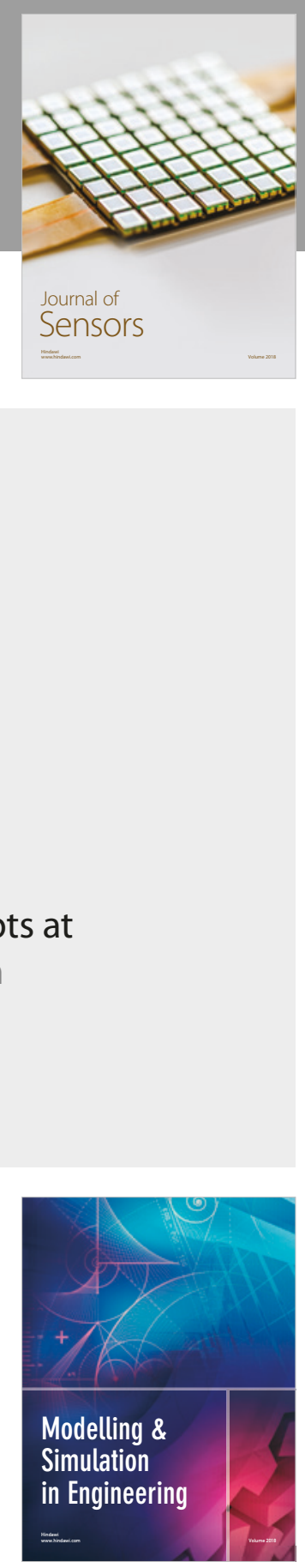

\section{Advances \\ Multimedia}
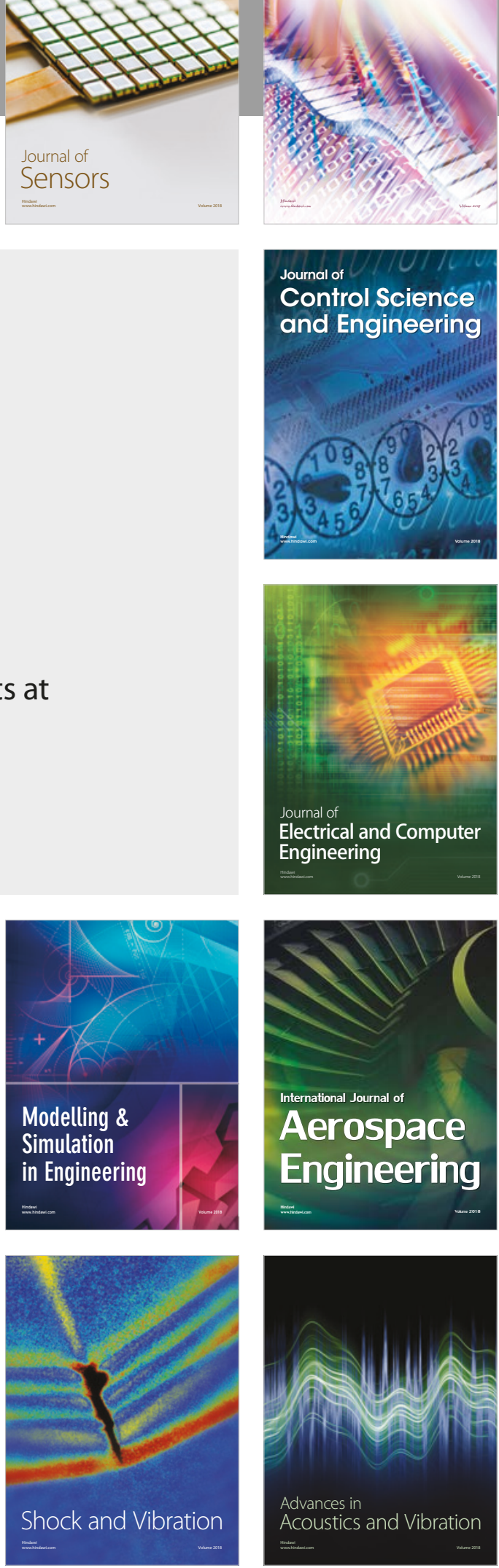